\title{
On the Economic Meaning of MACHINA'S FRÉCHET Differentiability Assumption*
}

\author{
Zvi Safra (Corresponding Author) \\ Faculty of Management, Tel Aviv University \\ Tel Aviv 69978. Israel \\ E-mail: safraz@post.tau.ac.il Fax: 972-3-640-7742 \\ and \\ Uzi Segal \\ Department of Economics, Boston College \\ 140 Commonwealth Ave. Chestnut Hill MA 02467 \\ E-mail: segalu@bc.edu
}

October 11, 2001

\begin{abstract}
This note shows that Machina's (1982) assumption that preferences over lotteries are smooth has some economic implications. We show that Fréchet differentiability implies that preferences represent second order risk aversion (as well as conditional second order risk aversion). This implies, among other things, that decision makers buy full insurance only at the absence of marginal loading. We also show that with constant absolute and relative risk aversion, expected value maximization, second order risk aversion, and Fréchet differentiability are equivalent. Journal of Economic Literature Classification Number: D81.
\end{abstract}

*We thank Edi Karni and especially Mark Machina for their comments. Uzi Segal thanks the Social Science and Humanities Research Council of Canada for financial support. 


\section{Introduction}

Machina [6] introduced the concept of smooth representations of preferences over risky prospects. The real valued functional $V$ over distribution functions is Fréchet differentiable at $F$ if, for all $G$ in a neighborhood of $F$, there is a "local utility" function (over outcomes) $u(\cdot ; F): \mathbb{R} \rightarrow \mathbb{R}$ such that

$$
V(G)-V(F)=\int u(x ; F) d[G(x)-F(x)]+o(\|G-F\|)
$$

where $\|\cdot\|$ is the $L_{1}$-norm. The functional $V$ is Fréchet differentiable if it is Fréchet differentiable at all $F$ (see Zeidler [13]). The preference relation $\succeq$ is called Fréchet differentiable if it has a Fréchet differentiable representation. Machina argued that since many problems in economics involve only local analysis (for example, optimization and comparative statics analysis), and since the functional $V$ can be locally approximated by an expected utility functional, it should follow that many of the economic results of expected utility apply to all (smooth) nonexpected utility preferences.

It turns out that some well known preference relations are not Fréchet differentiable (see Chew, Karni, and Safra [1]), but they too have local utility approximations, although only in $L_{p}$ for $p>1$ (see Wang [11]). It thus seems that although the Fréchet differentiability assumption rules out some models, it does not have any effect on our ability to analyze local behavior under risk. In this note we show that this, however, is not true, and assuming Fréchet differentiability has some economic meaning.

\section{Orders of Risk Aversion and Fréchet Dif- ferentiability}

At points (i.e., degenerate distributions) where the increasing utility function $u: \mathbb{R} \rightarrow \mathbb{R}$ is differentiable (that is, almost everywhere), the expected utility functional $\int u(x) d F(x)$ behaves locally like expected value. Extending this property to nonexpected utility preferences, Segal and Spivak [9] defined the concept of second order risk aversion as follows. Consider the set $\mathcal{X}$ of all real bounded random variables on a measure space $\Omega=(S, \Sigma, P)$. For each $X \in \mathcal{X}$, let $F_{X}$ denote the cumulative distribution function of $X$. Without abuse of notation, we denote by $a$ the constant random variable with the 
value $a$ and its distribution function is denoted by $\delta_{a}$. Let $\succeq$ be a complete, transitive, continuous (with respect to the weak topology) and monotone (with respect to first order stochastic dominance) preference relation over $\mathcal{X}$. Monotonicity obviously implies that $X \sim Y$ whenever $F_{X}=F_{Y}$. For a random variable $\tilde{x}$ with expected value zero and for $x^{*} \in \mathbb{R}$, define the risk premium function $\pi$ implicitly by $\delta_{x^{*}-\pi\left(x^{*}, \tilde{x}\right)} \sim F_{x^{*}+\tilde{x}}$. Assume throughout that the preference relation $\succeq$ exhibits risk aversion with respect to mean preserving spreads. This preference relation is said to satisfy second order risk aversion at $x^{*}$ if for every such $\tilde{x}$,

$$
\left.\frac{\partial}{\partial t} \pi\left(x^{*}, t \tilde{x}\right)\right|_{t=0^{+}}=0 \quad \text { and }\left.\quad \frac{\partial^{2}}{\partial t^{2}} \pi\left(x^{*}, t \tilde{x}\right)\right|_{t=0^{+}}>0
$$

$(t \tilde{x}$ is the random variable that is obtained from $\tilde{x}$ by multiplying its outcomes by $t$ ). Similarly, the preference relation $\succeq$ is said to satisfy first order risk aversion at $x^{*}$ if for every non trivial $\tilde{x}$ with expected value zero,

$$
\left.\frac{\partial}{\partial t} \pi\left(x^{*}, t \tilde{x}\right)\right|_{t=0^{+}}>0
$$

First order risk aversion implies kinked indifference curves along the main diagonal in a states-of-the-world representation, while second order risk aversion implies smooth such indifference curves (see Segal and Spivak [9]). The concept of orders of risk aversion has some economic applications. A second order risk averter will buy full insurance if and only if its price is "fair," that is, when the price of a dollar insurance equals the probability of loss. On the other hand, a first order risk averter will buy full insurance even at the face of some marginal loading. (For this, see Segal and Spivak [9]. See also Karni [4] for other results concerning insurance and orders of risk aversion. For applications to the equity premium puzzle, see Epstein and Zin [2]).

Machina [7] offers an extensive discussion of different possible kinks of indifference curves in the space of payoffs. Some of these kinks are consistent with Fréchet differentiability (e.g., expected utility with a non differentiable utility function), and some are not (e.g., the rank dependent model). The difference between his approach and ours is that he is interested in the locally separable versus locally nonseparable nature of such kinks, while we are interested in the relation between Fréchet differentiability and orders of risk aversion. 
Segal and Spivak [10] show that under the assumption of Fréchet differentiability of the representation functional $V$, the preference relation $\succeq$ satisfies first [second] order risk aversion at a point $x^{*}$ iff the local utility $u\left(x ; \delta_{x^{*}}\right)$ is not differentiable [differentiable] with respect to its first argument at $x=x^{*}$. Using these results, Theorem 1 below states a connection between Fréchet differentiability and orders of risk aversion.

Theorem 1 If the preference relation $\succeq$ can be represented by a Fréchet differentiable functional $V$, then for almost all $x^{*}, \succeq$ satisfies second order risk aversion at $x^{*}$. In other words, the set of points where the preference relation $\succeq$ satisfies first order risk aversion is of measure zero.

Proof By monotonicity, the functional $V$ satisfies $V\left(\delta_{x}\right)>V\left(\delta_{y}\right) \Longleftrightarrow x>$ $y$, hence the set of points where $\partial V\left(\delta_{x}\right) / \partial x$ does not exist is of measure zero. The conclusion now follows from the equivalence of the following three conditions.

1. The derivative $\partial V\left(\delta_{x}\right) / \partial x$ exists at $x=x^{*}$.

2. The preference relation $\succeq$ satisfies second order risk aversion at $x=x^{*}$.

3. The local utility $u\left(x ; \delta_{x^{*}}\right)$ is differentiable with respect to its first argument at $x=x^{*}$.

The equivalence of (2) and (3) is proved in [10]. To see why (1) and (3) are equivalent, note that

$$
V\left(\delta_{x^{*}+\varepsilon}\right)-V\left(\delta_{x^{*}}\right)=u\left(x^{*}+\varepsilon ; \delta_{x^{*}}\right)-u\left(x^{*} ; \delta_{x^{*}}\right)+o(\varepsilon)
$$

(see eq. 1). Divide both sides by $\varepsilon$ and let $\varepsilon \rightarrow 0$ to obtain that $V\left(\delta_{x}\right)$ is differentiable with respect to $x$ at $x=x^{*}$ iff $u\left(x ; \delta_{x^{*}}\right)$ is differentiable with respect to its first argument at $x=x^{*}$.

The theorem implies that all models that have kinked indifference curves at all points along the main diagonal in a states-of-the-world representation are not $\left(L_{1}\right)$ Fréchet differentiable. Such is the rank dependent model (for a direct proof that this model is not Fréchet differentiable, see Chew, Karni and Safra [1]. For a proof that this model satisfies first order risk aversion, see Segal and Spivak [9]). Another example is Gul's [3] disappointment aversion 
model (see [5, Lemma 1]). Theorem 1 also implies that there is an economic behavioral difference between preference relations that are $L_{1}$ Fréchet differentiable and preferences that are $L_{p}$ Fréchet differentiable for $p>1$, as the latter may display first order risk aversion everywhere.

\section{Second Order Behavior and Nondifferen- tiability}

An interesting question is whether the converse of Theorem 1 holds. That is, whether a preference relation that satisfies second order risk aversion almost everywhere is necessarily Fréchet differentiable. In this section we offer an example showing that such a connection does not necessarily exist. In the sequel, we use the fact that expected utility with a differentiable utility $u$ exhibits second order risk aversion (see Segal and Spivak [9]).

Example 1 Let the set of outcomes be [0,2], let $u(x)=-x^{2}+5 x-3$, and define $F \succeq G$ iff $V(F) \geqslant V(G)$, where for every $F, V(F)=\min \{\mathrm{E}[F], \mathrm{E}[u(F)]\}$. We show that this preference relation satisfies second order risk aversion, but is not Fréchet differentiable.

Second order risk aversion: It is easy to verify that for $x<1, u(x)<x$, and for $x>1, u(x)>x$. Let $x^{*} \notin\{0,1,2\}$, say $0<x^{*}<1$. Then for every $\tilde{x} \neq \delta_{0}$ with expected value zero and for every sufficiently small $t>0$, $\mathrm{E}\left[u\left(x^{*}+t \tilde{x}\right)\right]<\mathrm{E}\left[x^{*}+t \tilde{x}\right]=x^{*}$. Second order behavior thus follows from the properties of the expected utility functional $\mathrm{E}[u(F)]$. A similar argument holds for the case $1<x^{*}<2$.

Next we show second order behavior at $x^{*}=1$. Since $u$ is a concave function, it follows that for all $\tilde{x}$ with zero expected value and for all $t>0$,

$$
\mathrm{E}\left[u\left(x^{*}+t \tilde{x}\right)\right]<u\left(x^{*}\right)=1=\mathrm{E}\left[x^{*}+t \tilde{x}\right]
$$

Also, for all $\pi>0, u\left(x^{*}-\pi\right)<x^{*}-\pi$. Therefore, both $x^{*}+t \tilde{x}$ and $x-\pi$ are computed with respect to $u$, which exhibits second order behavior.

Fréchet differentiability: Suppose $\succeq$ can be represented by the Fréchet differentiable functional $W(F)=h(V(F))$, where $h$ is strictly increasing. Let $\mathcal{F}_{1}=\{F: \mathrm{E}[F] \leqslant \mathrm{E}[u(F)]\}$, and let $\mathcal{F}_{2}=\{F: \mathrm{E}[F] \geqslant \mathrm{E}[u(F)]\}$. By 
the linearity of the expected utility functional, if $F, F^{\prime} \in \mathcal{F}_{i}$, then for all $\alpha \in[0,1], \alpha F+(1-\alpha) F^{\prime} \in \mathcal{F}_{i}$. Moreover,

$$
\frac{d}{d \alpha} V\left((1-\alpha) F+\alpha F^{\prime}\right)= \begin{cases}\mathrm{E}\left[F^{\prime}\right]-\mathrm{E}[F] & F, F^{\prime} \in \mathcal{F}_{1} \\ \mathrm{E}\left[u\left(F^{\prime}\right)\right]-\mathrm{E}[u(F)] & F, F^{\prime} \in \mathcal{F}_{2}\end{cases}
$$

Since $W$ is Fréchet differentiable, it follows that $\frac{d}{d \alpha} W\left((1-\alpha) F+\alpha F^{\prime}\right)$ exists for $F, F^{\prime} \in \mathcal{F}_{i}, i=1,2$, and is given by $h^{\prime}\left(V\left((1-\alpha) F+\alpha F^{\prime}\right)\right) \frac{d}{d \alpha} V((1-$ $\left.\alpha) F+\alpha F^{\prime}\right)$. Since this is true for all $F$ and $F^{\prime}$ in $\mathcal{F}_{i}$, we obtain that $h$ is everywhere differentiable. Next we show that there exists $F \in \mathcal{F}_{1} \cap \mathcal{F}_{2}$ such that $h^{\prime}(V(F))>0$.

Consider the set of lotteries

$$
\mathcal{F}_{3}=\left\{\frac{3}{4 x-x^{2}} \delta_{x}+\left(1-\frac{3}{4 x-x^{2}}\right) \delta_{0}: x \in[1,2]\right\}
$$

It is easy to verify that $\mathcal{F}_{3} \subset \mathcal{F}_{1} \cap \mathcal{F}_{2}$, and that $V\left(\mathcal{F}_{3}\right)=\left[1, \frac{3}{2}\right]$. Therefore, there exists $F \in \mathcal{F}_{3}$ such that $h^{\prime}$ at $V\left(F_{3}\right)$ is strictly positive.

Pick such a distribution $F$ and observe that since $W$ is Fréchet differentiable at $F$ and $h^{\prime}(V(F))>0$, then $V$ too is Fréchet differentiable at $F$. Define $G=\delta_{0} \in \mathcal{F}_{2}$ and $G^{\prime}=\delta_{2} \in \mathcal{F}_{1}$. Observe that $\frac{1}{2} G+\frac{1}{2} G^{\prime} \in \mathcal{F}_{2}$. Clearly, $V(G)=-3, V\left(G^{\prime}\right)=2$, and $V\left(\frac{1}{2} G+\frac{1}{2} G^{\prime}\right)=0$. We obtain the following derivatives.

$$
\begin{aligned}
& -\frac{d}{d \alpha} V((1-\alpha) F+\alpha G)=-3-\int u(x) d F(x)=-3-V(F) . \\
& \text { - } \frac{d}{d \alpha} V\left((1-\alpha) F+\alpha G^{\prime}\right)=2-\int x d F(x)=2-V(F) . \\
& \text { - } \frac{d}{d \alpha} V\left((1-\alpha) F+\alpha\left[\frac{1}{2} G+\frac{1}{2} G^{\prime}\right]\right)=-\int u(x) d F(x)=-V(F) .
\end{aligned}
$$

In other words, the derivative $\frac{d}{d \alpha} V\left((1-\alpha) F+\alpha F^{\prime}\right)$ is not linear in $F^{\prime}$. It thus follows that $V$ is not Fréchet differentiable. Since $W=h(V)$ and $h^{\prime}(V(F))$ exists, $W$ is not Fréchet differentiable at $F$. Also note that $V$, and hence $W$, are not even Gâteaux differentiable: A functional $V$ is Gâteaux differentiable at $F$ if for every $G, \delta V(F, G-F)$, which is given by

$$
\delta V(F, G-F)=\left.\frac{d}{d t} V((1-t) F+t G)\right|_{t=0^{+}}
$$


exists, and if it is a continuous linear function of $G-F . V$ is Gâteaux differentiable if it is Gâteaux differentiable at $F$ for every $F$ (see Zeidler [13, p. 191]).

\section{Conditional Second Order Behavior}

The original definitions of orders of risk aversion relate to mean preserving spreads from a nonstochastic outcome $x$. The standard analysis of risk aversion deals also with mean preserving spreads of a possible outcome in a lottery (see Rothschild and Stiglitz [8]). Likewise, it is possible to relate the concept of orders of risk aversion to such conditional spreads (see Loomes and Segal [5]).

Let $X=\left(x_{1}, p_{1} ; \ldots ; x_{n}, p_{n}\right)$, and let $\tilde{x} \neq \delta_{0}$ be a random variable with zero expected value. Define the conditional risk premium $\pi\left(X, x_{i}, t \tilde{x}\right)$ by

$$
\left(\ldots ; x_{i}-\pi\left(X, x_{i}, t \tilde{x}\right), p_{i} ; \ldots\right) \sim\left(\ldots ; x_{i}+t \tilde{x}, p_{i} ; \ldots\right)
$$

We say that the risk averse preference relation $\succeq$ satisfies conditional second order risk aversion for $X$ at $x_{i}$ if for every such $\tilde{x}$,

$$
\left.\frac{\partial}{\partial t} \pi\left(X, x_{i}, t \tilde{x}\right)\right|_{t=0^{+}}=0 \quad \text { and }\left.\quad \frac{\partial^{2}}{\partial t^{2}} \pi\left(X, x_{i}, t \tilde{x}\right)\right|_{t=0^{+}}>0
$$

Similarly, the preference relation $\succeq$ is said to satisfy first order risk aversion for $X$ at $x_{i}$ if for every non trivial $\tilde{x}$ with expected value zero,

$$
\left.\frac{\partial}{\partial t} \pi\left(X, x_{i}, t \tilde{x}\right)\right|_{t=0^{+}}>0
$$

In this section we show that Fréchet differentiability implies conditional second order risk aversion almost everywhere, but even this stronger concept does not imply Fréchet differentiability. (The reason Example 1 is nevertheless needed is explained below).

Theorem 2 If the preference relation $\succeq$ can be represented by a Fréchet differentiable functional $V$, then for all $X=\left(x_{1}, p_{1} ; \ldots ; x_{n}, p_{n}\right)$, for all $i$, and for almost all $x_{i}^{*}, \succeq$ satisfies conditional second order risk aversion for $\left(X_{-i}, x_{i}^{*}\right):=\left(x_{1}, p_{1} ; \ldots ; x_{i}^{*}, p_{i} ; \ldots ; x_{n}, p_{n}\right)$ at $x_{i}^{*}$. 
The proof is similar to the proof of Theorem 1.

The preference relation of Example 1 exhibits second order risk aversion. Moreover, using the same arguments, it exhibits conditional second order risk aversion everywhere. (As before, the only interesting case is when $\mathrm{E}[F]=\mathrm{E}[u(F)]$. Adding $t \tilde{x}$ with $\mathrm{E}[\tilde{x}]=0$ to $x_{i}$ will reduce $\mathrm{E}[u(F)]$, but will not change $\mathrm{E}[F]$. Also, since for all $x, u^{\prime}(x) \geqslant 1$, the computation of the conditional risk premium will be done with respect to $u$. Conditional second order thus follows from the fact that differentiable expected utility exhibits conditional second order risk aversion (see [5])). As we have shown above, for any representation of the preference relation of Example 1 there are points where this representation is not Fréchet (and Gâteaux) differentiable, but this set is small (the functional $V$ of this example is differentiable everywhere except for the set of distributions where $\mathrm{E}[F]=\mathrm{E}[u(F)]$. For any representation $W=h(V), h$ is almost everywhere differentiable, hence $W$ is not Fréchet differentiable only at the distributions where $V$ is nondifferentiable, or at the small sets of indifference sets where $h$ is nondifferentiable). The functional $V$ of the next example is nowhere Fréchet differentiable, moreover, given any representation $W=h(V)$ of the preference relation, there is a dense set of indifference sets such that for all points in these sets, $W$ is not Fréchet differentiable there (although it is Gâteaux differentiable). As we show, although this preference relation exhibits first order risk aversion everywhere, it exhibits conditional second order risk aversion almost everywhere.

Example 2 Here too the set of outcomes is [0,2], and the preference relation $\succeq$ can be represented by $V$ which is a Yaari [12] functional of the form $V(F)=\int x d g(F(x))$, where

$$
g(p)= \begin{cases}\frac{4 p}{3} & p \leqslant \frac{1}{2} \\ \frac{2 p+1}{3} & p>\frac{1}{2}\end{cases}
$$

This example exhibits first order risk aversion at all $x$. Indeed, let $\tilde{x}=$ $\left(x_{1}, p_{1} ; \ldots ; x_{n}, p_{n}\right)$ such that $x_{1} \leqslant \cdots \leqslant x_{n}$ and $\mathrm{E}(\tilde{x})=0$. Assume, wlg, that there exists $k$ such that $\sum_{i=1}^{k} p_{i}=\frac{1}{2}$. Clearly, $V\left(\delta_{x}\right)=x$, and $V\left(\delta_{x}+t \tilde{x}\right)=$ $x+\frac{2 t}{3} \sum_{i=1}^{k} p_{i} x_{i}$. Therefore, $\pi(x, t \tilde{x})=-\frac{2 t}{3} \sum_{i=1}^{k} p_{i} x_{i}$, and $\left.\frac{\partial}{\partial t} \pi(x, t \tilde{x})\right|_{t=0^{+}}=$ $-\frac{2}{3} \sum_{i=1}^{k} p_{i} x_{i}>0$. 
On the other hand, it is easy to verify that the preference relation represented by $V$ satisfies conditional second order risk aversion at $x_{i}$ unless $\sum_{j: x_{j}<x_{i}} p_{j}<\frac{1}{2}$, but $\sum_{j: x_{j} \leqslant x_{i}} p_{j}>\frac{1}{2}$. In other words, conditional second order risk aversion is satisfied everywhere, except for the outcome where the cumulative distribution function intersects the level $F(x)=\frac{1}{2}$.

Despite the fact that this preference relation is essentially (conditional) second order, the functional $V$ is nowhere Fréchet differentiable and for any transformation $W=h(V)$ of it, there is a dense set of indifference sets of $\succeq$ such that $W$ is not Fréchet differentiable at all distributions in these sets. Of course, we know already from Chew, Karni, and Safra [1] that the rankdependent functional $V$ (of which Yaari's dual theory is a special case) is not Fréchet differentiable at distributions $F$ that are discontinuous, but here we show that it is not differentiable at all distributions. The proof is tedious, and appears in the Appendix.

\section{Constant Risk Aversion}

A partial answer to the question whether second order risk aversion implies Fréchet differentiability is offered by the next proposition. If a preference relation $\succeq$ satisfies constant risk aversion (definition below) then having second order risk aversion almost everywhere (in fact, even at one point only), implies that $\succeq$ is the expected value preference relation, and hence Fréchet differentiable.

For $X \in \mathcal{X}$, let $\underline{X}$ be the lowest possible value of $X$ (that is, $\underline{X}$ is the supremum of the values of $x$ for which $\left.F_{X}(x)=0\right)$. Observe that for $X \in \mathcal{X}$ and $a>-\underline{X}, X+a \in \mathcal{X}$.

Definition 1 The preference relation $\succeq$ satisfies constant risk aversion if

1. For every $X$ and $Y$ and for every $a>\max \{-\underline{X},-\underline{Y}\}, X \succeq Y$ iff $X+a \succeq Y+a$; and

D. For every $X$ and $Y$ and for every $\lambda>0, X \succeq Y$ iff $\lambda X \succeq \lambda Y$.

Proposition 1 Assume constant risk aversion. Then the following three conditions are equivalent.

1. $V$ is an expected value functional. 
2. There is a wealth level $x^{*}$ at which the decision maker's preference relation displays second order risk aversion.

3. At all wealth levels, the decision maker's preference relation displays second order risk aversion.

Proof Obviously, (1) $\Longrightarrow(3) \Longrightarrow(2)$. By constant risk aversion, it is easy to verify that $(2) \Longrightarrow(3)$. To see why $(3) \Longrightarrow(1)$, pick $X=\left(x_{1}, p_{1} ; \ldots ; x_{n}, p_{n}\right)$ such that $\sum p_{i} x_{i}=x^{*}$, but $X \not \delta_{x^{*}}$. If $x$ is sufficiently close to $x^{*}$, then there is $\gamma \neq 0$ such that $X+\gamma=\left(x_{1}+\gamma, \ldots, x_{n}+\gamma\right) \sim \delta_{x^{*}}$. Define $\tilde{x}=\left(x_{1}-x^{*}, p_{1} ; \ldots ; x_{n}-x^{*}, p_{n}\right)$ and obtain by constant risk aversion that the risk premium the decision maker is willing to pay to avoid $t \tilde{x}$ is $t \gamma$. This contradicts the assumption that the preference relation satisfies second order risk aversion.

\section{Appendix: The Non-Differentiability of Exam- ple 2}

For a distribution function $F$ on $[0,2]$, denote by $\bar{F}$ the epigraph of $F, \bar{F}=$ $\{(x, p): p \geqslant F(x)\}$. Let $\mu$ be the Lebesgue measure on $[0,2] \times[0,1] \subset \mathbb{R}^{2}$. An alternative representation of the functional of Example 2 is given by

$$
V(F)=\frac{4}{3} \mu\left(\bar{F} \cap\left([0,2] \times\left[0, \frac{1}{2}\right]\right)+\frac{2}{3} \mu\left(\bar{F} \cap\left([0,2] \times\left[\frac{1}{2}, 1\right]\right)\right.\right.
$$

The local utility of Yaari's functional is given by (see Chew, Karni, and Safra [1])

$$
u(x ; F)=\int^{x} d g(F(y))
$$

In our case, let $x^{*}=\sup \left\{x: F(x) \leqslant \frac{1}{2}\right\}$. We obtain that

$$
u(x ; F)= \begin{cases}\frac{4 x}{3} & x \leqslant x^{*} \\ \frac{4 x^{*}}{3}+\frac{2}{3}\left(x-x^{*}\right) & x \geqslant x^{*}\end{cases}
$$

To show that $V$ is not Fréchet differentiable at $F$, we need to find $F_{n} \rightarrow F$ such that

$$
V\left(F_{n}\right)-V(F) \neq \int u(x ; F) d\left[F_{n}(x)-F(x)\right]+o\left(\left\|F_{n}, F\right\|\right)
$$


Assume first that $x^{*} \in(0,2)$, and that the variation of $F$ at $x^{*}$ is bounded away from zero and infinity (we define these requirements more precisely below). For a sufficiently large $n$, define $x_{n}^{1}=\sup \left\{x: F(x) \leqslant \frac{1}{2}+\frac{1}{\sqrt{n}}\right\}$ and $x_{n}^{2}=\sup \left\{x: F(x) \leqslant \frac{1}{2}-\frac{1}{\sqrt{n}}\right\}$. Let

$$
F_{n}^{1}(x)= \begin{cases}F(x) & x<x^{*}-\frac{1}{\sqrt{n}} \\ \frac{1}{2}+\frac{1}{\sqrt{n}} & x^{*}-\frac{1}{\sqrt{n}} \leqslant x<x_{n}^{1} \\ F(x) & x_{n}^{1} \leqslant x\end{cases}
$$

and

$$
F_{n}^{2}(x)= \begin{cases}F(x) & x<x_{n}^{2} \\ \frac{1}{2}-\frac{1}{\sqrt{n}} & x_{n}^{2} \leqslant x<x^{*}+\frac{1}{\sqrt{n}} \\ F(x) & x^{*}+\frac{1}{\sqrt{n}} \leqslant x\end{cases}
$$

$F_{n}^{1}$ is the minimal distribution above $F$ going through $\left(x^{*}-\frac{1}{\sqrt{n}}, \frac{1}{2}+\frac{1}{\sqrt{n}}\right)$ and $F_{n}^{2}$ is the maximal distribution below $F$ going through $\left(x^{*}+\frac{1}{\sqrt{n}}, \frac{1}{2}-\frac{1}{\sqrt{n}}\right)$ (see Fig. 1). Note that $V$ assigns the square $A_{n}^{2}=\left[x^{*}-\frac{1}{\sqrt{n}}, x^{*}\right] \times\left[\frac{1}{2}, \frac{1}{2}+\frac{1}{\sqrt{n}}\right]$ the weight $\frac{2}{3 n}$, while the weight of this square according to the local utility function is $\frac{4}{3 n}$. Similarly, $V$ assigns the square $A_{n}^{4}=\left[x^{*}, x^{*}+\frac{1}{\sqrt{n}}\right] \times\left[\frac{1}{2}-\frac{1}{\sqrt{n}}, \frac{1}{2}\right]$ the weight $\frac{4}{3 n}$, while its weight according to the local utility function is $\frac{2}{3 n}$. On the other hand, on the areas

- $A_{n}^{1,1}=\left\{(x, p): x \geqslant x^{*}, F(x) \leqslant p \leqslant F_{n}^{1}(x)\right\}$

- $A_{n}^{3,1}=\left\{(x, p): x \leqslant x^{*}, F(x) \leqslant p \leqslant \min \left\{F_{n}^{1}(x), \frac{1}{2}\right\}\right\}$

- $A_{n}^{1,2}=\left\{(x, p): x \geqslant x^{*}, \max \left\{F_{n}^{2}(x), \frac{1}{2}\right\} \leqslant p \leqslant F(x)\right\}$

- $A_{n}^{3,2}=\left\{(x, p): x \leqslant x^{*}, F_{n}^{2}(x) \leqslant p \leqslant F(x)\right\}$

the weights according to $V$ and $u(\cdot ; F)$ agree. Therefore, for $i=1,2$,

$$
d_{n}^{i}:=\left|\left(V\left(F_{n}^{i}\right)-V(F)\right)-\left(\int u(x ; F) d\left[F_{n}^{i}(x)-F(x)\right]\right)\right|=\frac{2}{3 n}
$$




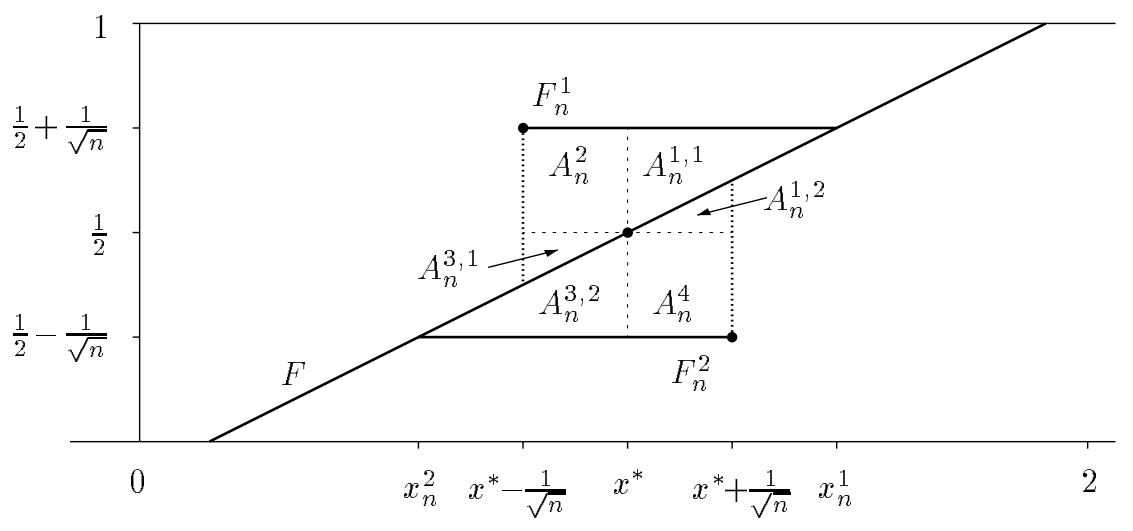

Figure 1: The distributions $F, F_{n}^{1}$, and $F_{n}^{2}$

We now show that $d_{n}^{i}$ is not $o\left(\left\|F_{n}^{i}, F\right\|\right)$ for either $i=1$ or $i=2$. Clearly, $\left\|F_{n}^{1}, F\right\|=\mu\left(A_{n}^{1,1} \cup A_{n}^{2} \cup A_{n}^{3,1}\right)$, while $\left\|F_{n}^{2}, F\right\|=\mu\left(A_{n}^{1,2} \cup A_{n}^{4} \cup A_{n}^{3,2}\right)$. If there are $0<K<L<\infty$ such that either

1. For every $n,\left[F\left(x_{n}^{1}\right)-F\left(x^{*}\right)\right] /\left(x_{n}^{1}-x^{*}\right)=\frac{1}{\sqrt{n}} /\left(x_{n}^{1}-x^{*}\right) \geqslant K$ and $\left[F\left(x^{*}\right)-F\left(x^{*}-\frac{1}{\sqrt{n}}\right)\right] /\left[x^{*}-\left(x^{*}-\frac{1}{\sqrt{n}}\right)\right]=\left[F\left(x^{*}\right)-F\left(x^{*}-\frac{1}{\sqrt{n}}\right)\right] / \frac{1}{\sqrt{n}} \leqslant L ;$ or

2. For every $n,\left[f\left(x^{*}\right)-f\left(x_{n}^{2}\right)\right] /\left(x^{*}-x_{n}^{2}\right)=\frac{1}{\sqrt{n}} /\left(x^{*}-x_{n}^{2}\right) \geqslant K$ and $\left[F\left(x^{*}+\right.\right.$ $\left.\left.\frac{1}{\sqrt{n}}\right)-F\left(x^{*}\right)\right] /\left[\left(x^{*}+\frac{1}{\sqrt{n}}\right)-x^{*}\right]=\left[F\left(x^{*}+\frac{1}{\sqrt{n}}\right)-F\left(x^{*}\right)\right] / \frac{1}{\sqrt{n}} \leqslant L$,

then we are through. The reason is that in the first case, $\lim \mu\left(A_{n}^{1,1}\right) \leqslant$ $1 /(2 n K)$ and $\lim \mu\left(A_{n}^{3,1}\right) \leqslant L /(2 n)$. Since $\mu\left(A_{n}^{2}\right)=\frac{1}{n}$, it follows that in the limit, $d_{n}^{1}$ and $\left\|F_{n}^{1}, F\right\|$ are of the same order of magnitude. In the second case, we obtain in a similar way that in the limit, $d_{n}^{2}$ and $\left\|F_{n}^{2}, F\right\|$ are of the same order of magnitude. Moreover, the same analysis holds for the cases where for every $n$, at most one of $\frac{1}{\sqrt{n}} /\left(x_{n}^{1}-x^{*}\right)$ and $\left[F\left(x^{*}\right)-F\left(x^{*}-\frac{1}{\sqrt{n}}\right)\right] / \frac{1}{\sqrt{n}}$ is less than $K$, or when for every $n$, at most one of $\left[F\left(x^{*}+\frac{1}{\sqrt{n}}\right)-F\left(x^{*}\right)\right] / \frac{1}{\sqrt{n}}$ and $\left(x^{*}-x_{n}^{2}\right) / \frac{1}{\sqrt{n}}$ is larger than $L$.

The two remaining cases happen when $F$ is either (almost) flat at $x^{*}$, or (almost) vertical there (or $x^{*} \in\{0,2\}$ ). Formally, if either for all $K$, (wlg) for 
every $n, \frac{1}{\sqrt{n}} /\left(x_{n}^{1}-x^{*}\right)<K$ and $\left[F\left(x^{*}\right)-F\left(x^{*}-\frac{1}{\sqrt{n}}\right)\right] / \frac{1}{\sqrt{n}}<K$, or when for all $L$, (wlg) for every $n,\left[F\left(x^{*}+\frac{1}{\sqrt{n}}\right)-F\left(x^{*}\right)\right] / \frac{1}{\sqrt{n}}>L$ and $\left(x^{*}-x_{n}^{2}\right) / \frac{1}{\sqrt{n}}>L$. In the first case, redefine $A_{n}^{2}$ to be the rectangle $\left[x_{n}^{2}, x^{*}\right] \times\left[\frac{1}{2}, \frac{1}{2}+\frac{1}{\sqrt{n}}\right]$, and redefine $A_{n}^{4}$ to be $\left[x^{*}, x_{n}^{1}\right] \times\left[\frac{1}{2}-\frac{1}{\sqrt{n}}, \frac{1}{2}\right]$ to obtain once again that either $d_{n}^{1}$ is of the same order of magnitude as $\left\|F_{n}^{1}, F\right\|$, or $d_{n}^{2}$ is of the same order of magnitude as $\left\|F_{n}^{2}, F\right\|$. In the second case, redefine $A_{n}^{2}$ to be the rectangle $\left[x^{*}-\frac{1}{\sqrt{n}}, x^{*}\right] \times\left[\frac{1}{2}, F\left(x^{*}+\frac{1}{\sqrt{n}}\right)\right]$, and redefine $A_{n}^{4}$ to be $\left[x^{*}, x^{*}+\frac{1}{\sqrt{n}}\right] \times\left[F\left(x^{*}-\right.\right.$ $\left.\left.\frac{1}{\sqrt{n}}\right), \frac{1}{2}\right]$ for similar results.

The proof that any transformation $W=h(V)$ has a dense set of indifference sets such that $W$ is not Fréchet differentiable at all points in these sets is similar to that of Example 1. All we need to show is that in any open interval in the range of $W$ there is a point $w$ such that either $h$ is not differentiable at $h^{-1}(w)$, or its derivative there is not zero. 


\section{References}

[1] Chew, S.H., E. Karni, and Z. Safra, Risk aversion in the theory of expected utility with rank dependent probabilities, J. Econ. Theory 42 (1987), 370-381.

[2] Epstein, L.G. and S.E. Zin, 'First-order' risk aversion and the equity premium puzzle, J. Monetary Economics 26 (1990), 387-407.

[3] Gul, F., A theory of disappointment aversion, Econometrica 59 (1991), $667-686$.

[4] Karni, E., Non-expected utility and the robustness of the classical insurance paradigm: Discussion, Geneva Papers on Risk and Insurance Theory 20 (1995), 51-56.

[5] Loomes, G. and U. Segal, Observing orders of risk aversion, J. Risk and Uncertainty 9 (1994), 239-256.

[6] Machina, M.J., 'Expected utility' analysis without the independence axiom, Econometrica 50 (1982), 277-323.

[7] Machina, M.J., Payoff kinks in preferences over lotteries, mimeo, 2000.

[8] Rothschild, M. and J. Stiglitz, Increasing risk I: A definition, J. Econ. Theory 2 (1970), 225-243.

[9] Segal, U. and A. Spivak, First-order versus second-order risk aversion, J. Econ. Theory 51 (1990), 111-125.

[10] Segal, U. and A. Spivak, First-order risk aversion and nondifferentiability, Econ. Theory 9 (1997), 179-183.

[11] Wang, T., $L_{p}$-Fréchet differentiable preference and "local utility" analysis, J. Econ. Theory 61 (1993), 139-159.

[12] Yaari, M.E., The dual theory of choice under risk, Econometrica 55 (1987), 95-115.

[13] Zeidler, E., "Nonlinear Functional Analysis and its Applications," vol. III, Springer, New York, 1985. 